\title{
Characterization of Biodegradable Medical Materials
}

\author{
JAROSLAW W. DRELICH $\oplus^{1,2}$ \\ 1.-Department of Materials Science and Engineering, Michigan Technological University, Hou- \\ ghton, MI 49931, USA. 2.—e-mail: jwdrelic@mtu.edu
}

Since the twentieth century, the development of artificial implants needed in surgical restorative health care has been dominated by biologically inert and corrosion-resistant materials. These permanent implants either need to be removed through a secondary surgery or remain in the host body for a lifetime. Remaining implants often cause long-term complications either from slow but progressive corrosion and material properties deterioration or as a result of characteristics that are different from the intruded biological environment. The paradigm of permanent implants has been challenged over the last two decades through the development of biodegradable implant materials. Biodegradable implants can provide the biomechanical support necessary throughout the healing process but will eventually dissolve and be replaced by the host tissue. Biodegradable polymers were the primary candidates for implants, given the ease of processing and shaping, together with their predictable degradation products. However, their low mechanical properties, poor visibility in the body, and acidification of the implantation site during degradation have limited widespread clinical application of polymer implants. Biodegradable metallic materials have superior mechanical properties and have been investigated as candidate materials for vascular, orthopedic, suturing, and other medical applications since the beginning of the twenty-first century. However, new materials for medical applications must fulfill clinical requirements related to deployment, efficacy, and device safety, which pose challenges to metallurgists in designing, characterizing, and testing these materials, as discussed in the following collection of papers written by experts in the field of biodegradable implants.

Jaroslaw W. Drelich is a guest editor for the Materials Characterization Committee of the TMS Extraction \& Processing Division and the Biomaterials Committee of the TMS Functional Materials Division and the Light Metals Division. Dr. Drelich is the guest editor for the topic Characterization of Biodegradable Medical Materials in this issue.
The development of materials for bioabsorbable implants relies heavily on corrosion testing in pseudophysiological solutions, where a candidate material degrades faster than it does in the body. The quantitative differences between corrosion kinetics in a laboratory environment (in vitro) and in a body (in vivo) are largely unknown, but in vitro tests enable prescreening of materials regarding corrosion rate and formulation of corrosion products, with the ultimate goal of reducing dependence on animal studies. The paper titled "Investigating $\mathrm{Mg}$ Biocorrosion In Vitro: Lessons Learned and Recommendations," by Andrej Atrens' research team from Australia, outlines appropriate experimental approaches for conducting an initial evaluation of the in vitro degradation properties of $\mathrm{Mg}$ based biodegradable alloys. This contribution provides much needed, comprehensive guidelines for in vitro biocorrosion assessment of $\mathrm{Mg}$ alloys. The aim of the second study, described in the paper "Comparative Studies on Degradation Behavior of Pure Zinc in Various Simulated Body Fluids" and led by Yufeng Zheng from China, is to highlight the multifaceted degradation behavior of pure zinc as a function of test-solution composition based on standard static immersion testing and electrochemical measurements. The authors show that the chemical composition of the medium plays a fundamental role in the degradation pattern of pure $\mathrm{Zn}$ and that the synergy of chlorides, carbonates, phosphates, and complex organic molecules present in the body environment is a key factor affecting in vitro degradation of $\mathrm{Zn}$. Zinc and zinc alloys are the newest addition to the list of biodegradable metals for medical applications, after polymers and $\mathrm{Mg}$ - and $\mathrm{Fe}$-based materials. The rise of $\mathrm{Zn}$ alloys in medical applications is attributed to the paper published by my research team. ${ }^{1}$ Since that time, $\mathrm{Zn}$ alloys have become the next important class of materials, after Mg alloys, being explored for prototyping biodegradable medical implants. 
The next paper in this collection describes the formulation and characterization of new Mg-based alloys. "Mechanical and Corrosion Properties of Two Precipitation-Hardened Mg-Y-Nd-Gd-Dy Alloys with Small Changes in Chemical Composition," by Petra Maier et al. from Germany, discusses the mechanical properties and corrosion performance of two Mg-Y-Nd-Gd-Dy alloys, WE32 and WE33, with minor variation in chemical composition, in extruded, solution, and precipitation heat-treated conditions. The authors show that solution heat treatment facilitates grain growth but deteriorates strength integrity. Corrosion evaluations through potentiodynamic studies in Ringer acetate solution indicate that the corrosion rate depends strongly on the alloy and heat treatment condition, as well as on the test method.

The last two papers in this collection focus on in vivo animal studies, which are typically less available to metallurgists. In the paper "Preclinical In Vivo Evaluation and Screening of Zinc-Based Degradable Metals for Endovascular Stents," Jeremy Goldman and his team from Michigan Technological University again used their rat aorta metal wire implantation model, developed in 2012, to simulate the presence of a stent strut within the vascular space. The wire model, which is refined in this new contribution with adapted metrics to characterize the long-term progression of the neointima (scar tissue) forming around wire, allows for simplified investigations at the interface between the candidate metal and the arterial environment. This cost-effective approach allows researchers to characterize the material's corrosion behavior and biocompatibility in the absence of complex factors related to stent deployment and mechanical function. In the paper "The Interface between Degradable Mg and Tissue," Regine Willumeit-Römer from Germany describes the very challenging task of analyzing the "interface" and bonding between the implanted metal and the surrounding bone tissue. Biodegradable implants corrode over time in such a way that the interface between metal and tissue retreats, leaving extra space for the tissue to grow. However, at the same time, the surrounding tissue is exposed to more metal degradation by-products and the implant continues interaction with body fluids and biological constituents. As a result, the interface location, chemical composition (including $\mathrm{pH}$ ), and biological composition and histology of surrounding tissue are time dependent. This presents many challenges to research teams in this field, requiring expertise that goes beyond metallurgy and corrosion science.

The following papers are being published under the topic of Characterization of Biodegradable Medical Materials:

- "Investigating Mg Biocorrosion In Vitro: Lessons Learned and Recommendations" by Sean Johnston, Zhiming Shi, Jeffrey Venezuela, Cuie Wen, Matthew S. Dargusch, and Andrej Atrens

- "Comparative Studies on Degradation Behavior of Pure Zn in Various Simulated Body Fluids" by Xiao Liu, Hongtao Tang, Yang Liu, Pan Xiong, Hui Guo, Yan Cheng, Her-Hsiung Huang, and Yufeng Zheng

- "Mechanical and Corrosion Properties of Two Precipitation-Hardened Mg-Y-Nd-Gd-Dy Alloys with Small Changes in Chemical Composition" by Petra Maier, Nadine Lauth, Chamimi Mendis, Maximilian Bechly, and Norbert Hort

- "Preclinical In Vivo Evaluation and Screening of Zinc-Based Degradable Metals for Endovascular Stents" by Roger J. Guillory II, Alexander A. Oliver, Emma K. Davis, Elisha J. Early, Jaroslaw W. Drelich, and Jeremy Goldman

- "The Interface between Degradable $\mathrm{Mg}$ and Tissue" by Regine Willumeit-Römer

To download any of these papers, follow the url $\mathrm{h}$ ttp://ink.springer.com/journal/11837/71/4/page/1 to the table of contents page for the April 2019 issue (vol. 71, no. 4).

\section{REFERENCE}

1. P.K. Bowen, J. Drelich, and J. Goldman, Adv. Mater. 25, 2577 (2013).

Publisher's Note Springer Nature remains neutral with regard to jurisdictional claims in published maps and institutional affiliations. 